\title{
Interobserver variation in the reporting of cervical colposcopic biopsy specimens: comparison of grading systems
}

W G McCluggage, H Bharucha, L M Caughley, A Date, P W Hamilton, C M Thornton, M Y Walsh
Department of

Group of Hospitals

Trust, Belfast

W G McCluggage

H Bharucha

L M Caughley

A Date

P W Hamilton

C M Thornton

M Y Walsh

The Queen's

University of Belfast

H Bharucha

P W Hamilton

Correspondence to:

Dr W G McCluggage,

Department of Pathology,

Royal Group of Hospitals

Trust, Grosvenor Road,

Belfast BT12 6BL.

Accepted for publication 16 July 1996

\begin{abstract}
Aims-To assess interobserver variation in reporting cervical colposcopic biopsy specimens and to determine whether a modified Bethesda grading system results in better interobserver agreement than the traditional cervical intraepithelial neoplasia (CIN) grading system.

Methods-One hundred and twenty five consecutive cervical colposcopic biopsy specimens were assessed independently by six histopathologists. Specimens were classified using the traditional CIN grading system as normal, koilocytosis, CIN I, CIN II, or CIN III. The specimens were also classified using a modified Bethesda grading system as either normal, low grade squamous intraepithelial lesion (LSIL) or high grade squamous intraepithelial lesion (HSIL). Participants were also asked to categorise biopsy specimens by the CIN system with the addition of the recently proposed category "basal abnormalities of uncertain significance (BAUS)". The degree of agreement between participants was assessed by kappa statistics.

Results-Using the CIN system, interobserver agreement was generally poor: unweighted and weighted kappa values between individual pairs of observers ranging from 0.05 to 0.34 (average 0.20 ) and from 0.20 to 0.54 (average 0.36 ), respectively. With the modified Bethesda system, interobserver agreement was better but still poor: unweighted and weighted kappa values ranging from 0.15 to 0.58 (average 0.30 ) and from 0.21 to 0.61 (average 0.36), respectively. There was little or no agreement between observers in the diagnosis of BAUS.

Conclusions-Interobserver agreement in the reporting of cervical colposcopic biopsy specimens using the CIN grading system is poor. Agreement, while still poor, is better when a modified Bethesda grading system is used. There is little or no consensus in the diagnosis of BAUS. (f Clin Pathol 1996;49:833-835)
\end{abstract}

Keywords: cervical intraepithelial neoplasia, diagnosis, interobserver variation, Bethesda system.

Cervical intraepithelial neoplasia (CIN) is the preferred designation for the range of squamous intraepithelial abnormalities of the cervix that are associated with an increased risk of subsequent development of invasive squamous carcinoma. Traditionally, intraepithelial abnormalities are graded as CIN I, CIN II or CIN III depending on the degree of differentiation. Many cases of CIN are associated with infection by human papillomavirus (HPV), ${ }^{1}$ the histological hallmark of which is koilocytosis. This may be identified in cervical biopsy specimens with or without CIN. In addition to the typical changes of koilocytosis, HPV infection may result in morphological changes within cervical squamous epithelia which may be confused with CIN. Previous studies have demonstrated poor interobserver agreement in the grading of CIN and in the differentiation of low grades of CIN from koilocytosis. ${ }^{2}{ }^{3}$ In spite of these difficulties, histological assessment remains important in determining treatment, clinical management and subsequent follow up of patients with cervical squamous intraepithelial lesions.

It is also apparent that, analogous to the histological assessment of cervical squamous lesions, there is considerable interobserver variation in the cytological classification of cervical specimens. ${ }^{45}$ In the USA the traditional three tier CIN grading system used for the reporting of cervical cytology specimens has been replaced by a two tier grading system This system, known as the Bethesda system, was developed in 1988 following a workshop sponsored by the National Cancer Institute which addressed the standardisation of cervical cytopathology reports. ${ }^{6}$ Premalignant cervical squamous abnormalities were divided into low grade squamous intraepithelial lesions (LSIL), which encompass features of HPV infection and CIN I, and high grade squamous intraepithelial lesions (HSIL), which encompass CIN II and CIN III. The Bethesda system is deemed to be clinically relevant in that patient management, treatment and follow up is partly dependent on whether the lesion is high or low grade. Some, including the originator of the histological CIN grading system, have proposed that a modified Bethesda system be used for the histological grading of cervical squamous lesions. ${ }^{7}$ It is hoped that such a system, as well as being clinically relevant, migh improve diagnostic consistency.

In the UK a new category "basal abnormalities of uncertain significance (BAUS)" has been suggested as an addition to the traditional CIN classification. ${ }^{8}$ It might be anticipated that the introduction of an additional category at 
Table 1 Weighting factors used for weighted kappa assessment in CIN grading system

\begin{tabular}{llllll}
\hline & Normal & Koilocytosis & CIN I & CIN II & CIN III \\
\hline Normal & 0 & 1 & 2 & 3 & 4 \\
Koilocytosis & 1 & 0 & 1 & 2 & 3 \\
CIN I & 2 & 1 & 0 & 1 & 2 \\
CIN II & 3 & 2 & 1 & 0 & 1 \\
CIN III & 4 & 3 & 2 & 1 & 0 \\
\hline
\end{tabular}

the lower end of the spectrum of cervical squamous intraepithelial abnormalities would result in even greater disagreement among observers in the assessment of these lesions. This has been confirmed in a recent report. ${ }^{9}$ The purpose of this study was threefold: (1) to assess interobserver variation in the reporting of cervical colposcopic biopsy specimens using the CIN system; (2) to assess interobserver variation in the same biopsy specimens using a modified Bethesda grading system; and (3) to assess interobserver variation in reporting of the BAUS category.

\section{Methods}

Specimens used in the study comprised 125 consecutive cervical colposcopic biopsy specimens submitted to the Department of Pathology, Royal Group of Hospitals Trust, Belfast. Specimens were received in formalin, embedded in paraffin wax and stained with haematoxylin and eosin. Each biopsy specimen was assessed independently by six histopathologists. These comprised five consultants of three to 25 years standing and a senior registrar who has been in pathology for seven years. Two of the participants have an interest in gynaecological histopathology. Participants were supplied with the patient's age but were given no details as to clinical, colposcopic or cytological findings. A proforma (fig 1) was used to classify each biopsy specimen using the traditional CIN grading system (normal, koilocytosis, CIN I, CIN II, or CIN III) and using a modified Bethesda system (normal, LSIL, HSIL). Participants were also asked to classify each biopsy specimen using the CIN grading system with the addition of the category BAUS. Each participant was supplied with a copy of the article where the term BAUS was proposed and where definitions of this category are pro-

Table 2 Unweighted and weighted kappa values between each pair of observers for CIN and modified Bethesda grading systems

\begin{tabular}{|c|c|c|c|c|}
\hline \multirow{2}{*}{$\begin{array}{l}\text { Pairs of } \\
\text { observers }\end{array}$} & \multicolumn{2}{|l|}{ CIN system } & \multicolumn{2}{|l|}{ Bethesda system } \\
\hline & $\begin{array}{l}\text { Unweighted } \\
\text { kappa }\end{array}$ & Weighted kappa & Unweighted kappa & Weighted kappa \\
\hline $1 v 2$ & 0.16 & 0.32 & 0.16 & 0.25 \\
\hline $1 v 3$ & 0.24 & 0.47 & 0.40 & 0.46 \\
\hline $1 v 4$ & 0.21 & 0.38 & 0.28 & 0.37 \\
\hline $1 v 5$ & 0.34 & 0.54 & 0.58 & 0.61 \\
\hline $1 v 6$ & 0.13 & 0.23 & 0.18 & 0.23 \\
\hline $2 v 3$ & 0.23 & 0.40 & 0.34 & 0.41 \\
\hline $2 v 4$ & 0.17 & 0.31 & 0.24 & 0.31 \\
\hline $2 v 5$ & 0.19 & 0.37 & 0.21 & 0.28 \\
\hline $2 v 6$ & 0.34 & 0.46 & 0.45 & 0.51 \\
\hline $3 v 4$ & 0.27 & 0.44 & 0.37 & 0.45 \\
\hline $3 v 5$ & 0.27 & 0.46 & 0.40 & 0.46 \\
\hline $3 v 6$ & 0.13 & 0.24 & 0.22 & 0.28 \\
\hline $4 v 5$ & 0.17 & 0.38 & 0.30 & 0.37 \\
\hline $4 v 6$ & 0.07 & 0.20 & 0.17 & 0.22 \\
\hline $5 v 6$ & 0.05 & 0.25 & 0.15 & 0.21 \\
\hline Average value & 0.20 & 0.36 & 0.30 & 0.36 \\
\hline
\end{tabular}

vided. ${ }^{8}$ Cases of immature squamous metaplasia, where there was no associated koilocytosis or CIN, were to be classified as normal.

The degree of overall agreement between $c$ each pair of observers was evaluated by kappa $\Omega$ statistics. Kappa is an index of interobserver $\overline{\bar{J}}$ variation which has been corrected for chance 0 and indicates the degree of interobserver variation over and above that which would be expected by chance alone. ${ }^{10}$ The value of kappa $\underset{\overrightarrow{0}}{\overrightarrow{0}}$ can range from -1.0 to +1.0 . A value of 0 indicates chance agreement only, while a value of $\frac{\bar{\sigma}}{5}$ +1.0 indicates perfect agreement. It is gener- $\frac{\bar{m}}{T}$ ally accepted that a value of 0.75 or above $\mathbb{Q}$ reflects excellent agreement, a value of $0.4-\frac{0}{\omega}$ 0.75 suggests fair to good agreement and a $\overrightarrow{0}$ value of less than 0.4 means agreement is poor. Both unweighted and weighted kappa analysis was carried out. Weighted kappa takes into account the fact that some disagreements are more serious than others. ${ }^{11}$ In unweighted $\overrightarrow{0}$ analysis, a diagnostic disagreement of normal $\vec{\circ}$ and CIN III is treated in the same way as a diagnostic disagreement of koilocytosis and $\omega$ CIN I. In weighted kappa analysis this is 은 adjusted to ensure that larger differences in a ranked grading scheme will have a stronger influence on the final kappa value. In this study a linear weighting scheme was used as shown in table 1 . The values in table 1 indicate the $\vec{\bullet}$ weights applied when different combinations were encountered.

\section{Results}

Table 2 shows unweighted and weighted kappa values between each pair of observers for both the CIN grading system and the modified Bethesda grading system. With the CIN system, kappa values ranged widely from 0.05 to 0.34 with an average value of 0.20 . Agreement was poor between all pairs of observers. When weighted kappa was used, values ranged from 0.20 to 0.54 with an average value of 0.36 . Fair to good agreement (kappa greater than 0.4) was achieved between six of 15 pairs of observers. Agreement was no better between the two observers with an interest in gynaecological histopathology than between other pairs of observers.

With the modified Bethesda system, un- ज्ञ weighted kappa values between pairs of observ- $N$ ers were slightly better than with the CIN sys- N tem. Values ranged from 0.15 to 0.58 with an average of 0.30 . However, agreement was still 0 poor with only four of 15 pairs of observers achieving kappa values greater than 0.4 . ? Weighted kappa values with the Bethesda 0 system ranged from 0.21 to 0.61 with an average of 0.36 , the same as with the CIN system. Again, six of 15 pairs of observers achieved $\triangle$ kappa values greater than 0.4. Again, agreement was no better between the two observers with an interest in gynaecological histopathology than between other pairs of observers.

Tables 3 and 4 show the number of paired diagnoses with the CIN grading system and the modified Bethesda grading system, respectively. The number of occasions in which the category BAUS was used by each observer was $0,1,6,9,16$, and 48 . 
Table 3 Matrix showing number of paired observations between all pairs of participants in the CIN grading system

\begin{tabular}{llllll}
\hline Diagnosis & \multirow{2}{*}{ Normal } & Koilocytosis & CIN I & CIN II & CIN III \\
\hline Normal & 331 & 433 & 170 & 41 & 11 \\
Koilocytosis & 433 & 254 & 226 & 59 & 7 \\
CIN I & 170 & 226 & 78 & 76 & 32 \\
CIN II & 41 & 59 & 76 & 54 & 64 \\
CIN III & 11 & 7 & 32 & 64 & 39 \\
\hline
\end{tabular}

Table 4 Matrix showing number of paired observations between all pairs of participants in the modified Bethesda grading system

\begin{tabular}{llll}
\hline Diagnosis & Normal & LSIL & HSIL \\
\hline Normal & 331 & 601 & 49 \\
LSIL & 601 & 566 & 173 \\
HSIL & 49 & 173 & 155 \\
\hline
\end{tabular}

\section{Discussion}

The present study confirms that there is poor interobserver agreement in the reporting of cervical biopsy specimens and in the grading of cervical squamous intraepithelial lesions using the CIN grading system. This has been demonstrated in previous studies. ${ }^{2}{ }^{3}$ Unweighted kappa values between each pair of observers using this grading system were less than 0.4 . Weighted kappa values were also calculated to take account of the fact that some disagreements are more serious than others. Weighted kappa perhaps gives a truer picture than unweighted kappa as to the degree of interobserver variation. Using the CIN grading system, six of 15 pairs of observers achieved fair to good agreement. However, the average kappa value indicated that interobserver agreement was poor overall.

Table 2 shows that the areas which resulted in most disagreement were the separation of normal from koilocytosis, normal from CIN I and koilocytosis from CIN I. These observations are similar to those of previous studies, ${ }^{23}$ where disagreement was generally greater at the lower end of the spectrum. Although smaller numbers of high grade cases (CIN II and CIN III) were included in the study, it is apparent that there is difficulty in the separation of CIN II from CIN III and, in fewer cases, in the distinction of CIN I from CIN III. Surprisingly, there were also cases where difficulty existed in the distinction of CIN II and CIN III from normal cervical squamous epithelium. Retrospective review revealed that this difficulty most probably represented cases of immature squamous metaplasia being mistaken for higher grades of CIN, a recognised pitfall. In the present study, cases of immature squamous metaplasia, rather than being categorised separately, were classified as normal if there was no associated koilocytosis or CIN.

The Bethesda system for the reporting of cervical cytology specimens has gained widespread acceptance in the USA in recent years. In the present study, a modified histological version of the Bethesda system was used with koilocytosis or CIN I classified as LSIL and CIN II and CIN III classified as HSIL. It was expected that this grading system would result in a noticeable improvement in interobserver agreement. However, average weighted and unweighted kappa values were still less than 0.4 and the average weighted kappa of 0.36 was identical with that achieved using CIN grading. As can be seen from table 3, there was notable difficulty in the distinction of normal from LSIL and in the distinction of LSIL from HSIL.

In 1991, a Working Party convened by the British Society for Colposcopy and Cervical Pathology suggested that the traditional CIN grading system for histological reporting should be retained and proposed the introduction of a new category "basal abnormalities of uncertain significance (BAUS)". 8 The term BAUS was to be used in cases of basal cell changes that are not clearly CIN I but, equally, cannot be unequivocally allocated to a nonneoplastic category. Morphological definitions of this category were provided. A recent study revealed poor interobserver agreement in the classification of BAUS. ${ }^{9}$ This was confirmed in the present study where usage of this category varied from 0 to 48 occasions. On no occasion was there agreement on the usage of this category by more than two participants.

In conclusion, the study confirms noticeable interobserver variation in the histological classification of cervical biopsy specimens. Although interobserver agreement was better with the modified Bethesda grading system than the traditional CIN grading system, it was still poor. There was little agreement on usage of the BAUS category.

1 Herrington CS. Human papillomaviruses and cervical neoplasia. I. Classification, virology, pathology and epidemiology. $\mathcal{F}$ Clin Pathol 1994;47:1066-71.

2 Ismail SM, Colclough AB, Dinnen JS, Eakins D, Evans DMD, Gradwell E, et al. Observer variation in histopathological diagnosis and grading of cervical intraepithelia neoplasia. BMF 1989;298:707-10.

3 Robertson AJ, Anderson JM, Swanson Beck J, Burnett RA, Howatson SR, Lee FD, et al. Observer variability in histopathological reporting of cervical biopsy specimens. $f$ Clin Pathol 1989;42:231-8.

4 Seybolt JF, Johnson WD. Cervical cytodiagnostic problems: A survey. Am $\mathcal{F}$ Obstet Gynecol 1971;109:1089-103.

5 O'Sullivan JP, Ismail SM, Barnes WSF, Deery ARS, GradO'Sullivan JP, Ismail SM, Barnes WSF, Deery ARS, Grad-
well E, Harvey JA. Inter- and intraobserver variation in the reporting of cervical smears: specialist cytopathologists versus histopathologists. Cytopathology 1996;7:78-89.

6 Solomon D. The 1988 Bethesda system for reporting cervical/vaginal cytological diagnoses. Acta Cytol 1989;33: 567-74.

7 Richart RM. A modified terminology for cervical intraepithelial neoplasia. Obstet Gynecol 1990;75:131-3.

8 Anderson MC, Brown CL, Buckley CH, Fox H, Jenkins D, Love $\mathrm{D}$, et al. Current views on cervical intraepithelial neoplasia. F Clin Pathol 1991;44:969-78.

9 Creagh T, Bridger JE, Kupek E, Fish DE, Martin-Bates E, Wilkins MJ. Pathologist variation in reporting cervical borderline epithelial abnormalities and cervical intraepithelia neoplasia. F Clin Pathol 1995;48:59-60.

10 Fleiss JL. Statistical methods for rates and proportions. 2nd edn. New York: Wiley, 1981:212-34.

11 Fleiss JL, Cohen J. The equivalence of weighted kappa and intraclass correlation coefficient as measures of reliability. Educational Psychol Measurements 1973;33:613-19. 